The form of comments of course raises many questions: Which forms will be accepted? Who decides that a comment is suitable? Will some action be taken to resolve conflicting opinions? Will spontaneous refereeing be sufficient, or will it still be necessary to solicit evaluation, as in the traditional system? The evaluation, to be useful, has to be easily accessible, and this affects both the precise form of the evaluation, and the electronic tools needed to retrieve it (can one think here in terms of a Michelin guide for articles in say quantum gravity?).

It is often said that in the traditional system, interaction between referees and authors leads to improved articles whereas in the new system this interaction will disappear. First, from my own editorial experience, this improvement effect should not, unfortunately, be overestimated. Moreover, since authors will slowly discover that it is useless to accumulate unreadable (and therefore unread) articles, they will be subject to a renewed pressure to write better articles, an effect which in the long run may more than offset the temporary inconvenience of having no interaction.

Secondly, the logic of the new system requires comments to be signed, leading to the disappearance of the review system based on anonymous referees which has some merit. Whether this will have any significant impact is unclear. But whatever the ultimate form of the evaluation and commenting process, I believe the electronic medium opens up new and exciting possibilities which will eventually be of tremendous importance for the scientific community.

\section{ACKNOWLEDGEMENT}

Although it does not necessarily reflects all his views, this contribution has benefitted from many interesting discussions with Paul Ginsparg from the Los Alamos National Laboratory, USA.

\title{
Reflecting an Enlarged Community
}

\section{EUROPEAN JOURNAL OF PHYSICS}

\section{Samuelsson (Linköping University) took over late last year from A.B. Pippard as the Editor of the European Journal of Physics.}

The European Journal of Physics (EJP) is a relatively new journal which publishes articles destined for university-level lecturers. So articles must be neither too long nor too theoretical. However, it has been found that many university staff are unaware of the existence of this very useful journal. It is therefore important to publicise the journal more widely, and every effort will be made to do this. It should also be possible to make the journal more attractive by introducing new subjects, notably in experimental physics. But this needs to be discussed first by the Editorial Board, whose number will be brought up to the full complement of 15 by new appointments made by EPS Executive Committee. Proposals for the Board members now come the new EPS Interdivisional Group for Physics Education which members of all national societies can join. This reflects the importance of physics education

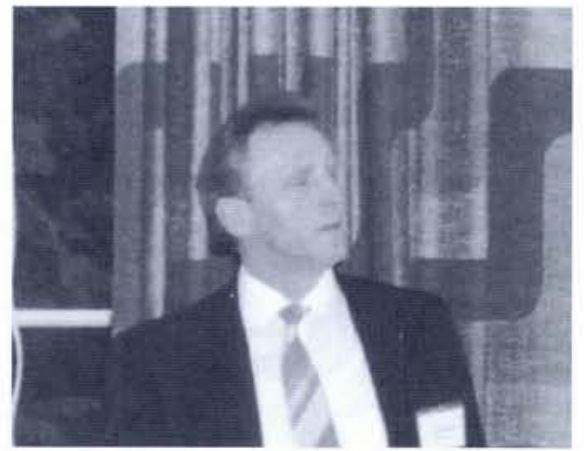

L.Samuelsson, the Editor of the European Journal of Physics, speaking at the 1995 EPS Council.

in EPS - a feature that EJP will definitely aim to highlight.

\section{EUROPHYSICS LETTERS}

R. Balian handed over the position of Editor-in Chief of Europhysics Letters to F.A. Gianturco (Chemistry Department, University of Rome) on 1 April.

I must first thank the European Physical Society for having allotted me a responsibility which has been extremely rewarding. My task as Editor-in-Chief of Europhysics Letters (EPL) has certainly been easier than that of my predecessors, N. Kurti and W. Buckel. It was they who had to set up a new journal - actually the only scientific journal (together with Astronomy \& Astrophysics) which is published under the simultaneous auspices of a large group of national societies. It was a pleasure for me to continue their work and to help in developing an enterprise which is exemplary in the construction of a "Europe of Science". Since its creation in 1986, EPL has reached a steady position: the number of subscribers is $\approx 900$, and the journal publishes $\approx 450$ articles p.a.
The quality of our journal relies mainly on its Co-editors. Their competence and devotion are certainly major elements of its success, and I wish here to express especially the gratitude of our community to them. One of their main difficulties is to coordinate the work of the referees. They have succeeded these last years in shortening the delay to acceptance, which now has a median of eight weeks for manuscripts that do not need revision (16 weeks with revision). This is still too long, but our rejection rate $(41 \%$ of the submitted manuscripts in 1994 - see table) and our revision rate $(80 \%$ of the accepted Letters) indicate the value of the work of the referees and Co-editors.

It has also been a pleasure for me to work with our efficient editorial office, and the technical teams should also be praised. Considering that EPL is edited in Geneva, composed in Bologna, printed in the west of France, and dispatched from near Paris, the present delay of only 5 to 6 weeks between the acceptance of an article and its publication is a remarkable achievement of our European cooperation.

As regards our Management Board, I shall give only one, very recent, example of the importance of its contribution. It has recently been decided that, from next July on, the titles and abstracts of all Letters will be available free-of-charge on World-Wide Web (http://www.edphy.fr/epl) as soon as they are accepted. This step, which should be appreciated by all physicists, reflects a permanent concern for modernization.

Although the situation thus looks rather satisfactory, the collective effort of our community should be used to remedy two distortions. First, the geographic distributions of authors submitting manuscripts and of subscribers does not yet fully reflect the relative importance of physics in various countries. This originates, of course, from some ancient traditions; but we feel that, at a time that the EPS is enlarging its audience, physicists from all over Europe should regard $E P L$, the journal run by our Society, as their privileged means for communicating new ideas and results in the form of Letters.

Second, more than one-half of the articles that we publish are somehow related to condensed matter or to statistical physics. Here again, historical reasons explain the distortion - a distortion which has some advantages. Nevertheless, the vocation of EPL is more universal since it is intended to include important and novel results which not only satisfy the specialist but are also of interest to a rather broad community. Research is often threatened by overspecialization: everyone is so busy in a particular field and often does not always realize the interest, in the long run, of a wide scientific culture. However, many discoveries were made by transposing an idea from one field to another. With this in mind, EPL is open to physicists from all fields: we urge them to submit manuscripts in domains where our journal is not yet well established, especially 